ORIGINAL

\title{
Prevalencia de neoplasias en caninos en la universidad de los Llanos, durante 2004 a 2007
}

\author{
Prevalence of neoplasm in canines in the university of the \\ Llanos, during 2004 to 2007
}

\begin{abstract}
Diana Bravo T, ${ }^{1}$ MVZ, Pablo Cruz-Casallas,${ }^{2}$ Ph.D, Julieta Ochoa A, ${ }^{*}$ M.Sc.
Universidad de los Llanos, Facultad de Ciencias Agropecuarias y Recursos Naturales, ${ }^{1}$ Escuela de Medicina Veterinaria y Zootecnia, ${ }^{2}$ Instituto de Acuicultura, Villavicencio, Meta, Colombia *Correspondencia: julieta.ochoa@gmail.com.
\end{abstract}

Recibido: Abril 10 de 2009; Aceptado: Octubre 20 de 2009.

\section{RESUMEN}

Objetivo. Describir y clasificar las enfermedades neoplásicas diagnosticadas en el laboratorio de Patología Veterinaria de la Universidad de los Llanos, desde enero de 2004 hasta junio de 2007. Materiales y métodos. Como fuente de información se utilizó la base de datos de las historias clínicas y de los exámenes de necropsia de la Clínica Veterinaria de la Universidad de los Llanos. Las muestras analizadas procedieron de especímenes obtenidos por biopsias y de tumores hallados durante necropsia. Se analizaron 322 casos clínicos, de los cuales 113 correspondieron a enfermedades neoplásicas (35\%). Resultados. En general, la especie más afectada fue la canina (93 de 113 casos), siendo las razas mestizas las más afectadas $(p<0.05)$ y el sistema orgánico más lesionado el cutáneo, con 68 casos $(60.2 \%)$, seguido de la glándula mamaria con 13 casos (11.5\%). Las neoplasias de mayor incidencia fueron carcinoma escamocelular (15 casos), tricoepitelioma (11 casos) y mastocitoma canino III (10 casos). Se observó una relación positiva entre la edad y la presentación de tumores $(p<0.01)$; el sexo no fue un factor determinante para la presentación de la enfermedad $(p>0.05)$. Conclusiones. Los tumores de piel fueron los de mayor incidencia, quizá por la mayor facilidad de diagnóstico durante la consulta clínica, comparados con otras neoplasias que afectan órganos y tejidos más profundos.

Palabras clave: Caninos, especie, incidencia, neoplasias. 


\section{ABSTRACT}

Objective. Describe and classify neoplastic diseases diagnosed at the Veterinary Pathology Laboratory at the University of the Llanos, between 2004 and 2007. Materials and methods. As a source of information was used database of medical records and autopsy examinations of the Veterinary Clinic of the University of the Llanos. The samples came from specimens obtained by biopsies and tumors found during necropsy. We analyzed 322 clinical cases, of which 113 were for cancer diseases (35\%). Results. In general, the most affected species was the dog (93 of 113 cases $)$, mixed races being the most affected $(p<0.05)$ and the organic system more injured skin, with 68 cases $(60.2 \%)$, followed by the gland breast with 13 cases $(11.5 \%)$. The highest incidence of neoplasms were: squamous cell carcinoma (15 cases), trichoepithelioma (11 cases) and canine mastocytoma III (10 cases). There was a positive relationship between age and presentation of tumors $(p<0.01)$, sex was not a determining factor for the presentation of the disease $(p>0.05)$. Conclusions. The skin tumors were the most impact, perhaps because of the ease of diagnosis during the clinical consultation, compared with other neoplasms that affect deeper organs and tissues.

Key words: Canine, species, incidence, neoplasms.

\section{INTRODUCCIÓN}

Entre los tumores epiteliales malignos se encuentra el carcinoma epidermoide o escamocelular, el cual es una neoplasia de bajo grado de malignidad, derivada de los epitelios escamosos estratificados. No poseen pigmentación ni papilación y constituyen el tumor más frecuente en animales, particularmente en caninos y felinos mayores de 5 años de edad (1). Es una patología asociada con la exposición a radiación ultravioleta, alquitrán, anilinas o tabaco (2). Su aspecto macroscópico es variable, pudiendo ser nodular, proliferativo, ulcerativo o costroso. Histopatológicamente, se extiende desde la epidermis, la cual aparece hiperplásica y ulcerada, hasta la dermis, formando cordones e islas de células escamosas con perlas de queratina; también hay disqueratosis intercelular, membrana basal disrupta, pleomorfismo nuclear y actividad mitótica $(2,3)$.

Otra neoplasia benigna de origen epitelial es el tricoepitelioma, el cual se desarrolla de las células indiferenciadas germinativas del folículo piloso. Microscópicamente consiste de múltiples quistes de tamaño variable, recubiertos por epitelio escamoso estratificado, llenos de queratina, dentro de los cuales están las células fantasma, que semejan células indiferenciadas del bulbo piloso. Presenta nidos de células basaloides con diferenciación folicular, con bordes indistintos y presencia de pequeñas cantidades de citoplasma fibrilar eosinofílico; núcleo oval a redondo, cromatina dispersa y nucléolo indistinto. Las células basales proliferan y maduran desde las capas externas del quiste, las cuales se caracterizan por la transición directa de células queratinizadas a células escamosas basales, con gránulos queratohilainos y núcleos descoloridos (4).

Entre los tumores mesenquimales malignos, el mastocitoma es el tumor de piel más común en perros adultos. Varias razas de caninos padecen de mastocitomas, entre ellas la Bóxer, Weimaraner, Golden retrievers, Pugs y Labradores, siendo estas dos últimas las más afectadas. La raza Shar-pei es más susceptible a desarrollar mastocitomas, tumores de alto grado de malignidad sin 
predilección de sexo (5). Su malignidad varía desde casi benignos, hasta extremadamente agresivos y metastizantes, afectando nódulos linfáticos regionales, hígado y bazo, pero raramente pulmones. Las células tumorales tienen forma redondeada, núcleo oval o redondo, basófilo y de posición central o paracentral; el citoplasma cuenta con numerosos gránulos que almacenan sustancias que caracterizan la función de estas células (6). Según la clasificación propuesta por Patnaik et al (7), existen tres grados de malignidad: Grado I, II y III.

En caninos, después de la piel, la glándula mamaria es el órgano más afectado por tumores. Entre las neoplasias más frecuentes se reporta el carcinoma simple, que tiene una fuerte tendencia a infiltrase en los tejidos y vasos circundantes. La cantidad de estroma es considerable y los linfocitos peri tumorales son comunes asociados a necrosis. Con base en su diferenciación y conducta biológica, puede ser clasificado por incremento en malignidad en: tubulopapilar, sólido o anaplásico (4).

El carcinoma complejo de glándula mamaria es relativamente común en el perro; es usualmente lobulado con componentes epiteliales y mioepiteliales. El componente epitelial puede clasificarse en tubulopapilar o sólido. La sustancia mucoide, ocasionalmente encontrada en estos tumores, permite ser diferenciada de cartílago joven y de carcinosarcomas, estos últimos caracterizados por la presencia de células embebidas en lagunas de matriz de cartílago (4).

El carcinosarcoma de glándula mamaria no es común en el perro. Este es un tumor compuesto de células que morfológicamente semejan componentes epiteliales malignos y sarcomatosos, como hueso o cartílago, los cuales pueden originarse de focos metaplásicos del componente epitelial o del estroma. Los tumores mixtos malignos tienen un mal pronóstico y hacen metástasis en el primer año de desarrollo (4).
Los tumores de hígado, como el carcinoma hepatocelular, afecta numerosas especies animales incluyendo gatos, perros, ovejas, cerdos, aves, truchas y vacas, siendo los perros la especie más afectada (8). La edad de los animales afectados oscila entre 10 y 11 años, aunque puede presentarse en animales menores de 4 años. Son neoplasias nodulares o difusas, como masas solitarias o involucrando varios lóbulos del hígado (4). Las metástasis ocurren primariamente a los pulmones, bazo, riñones, huesos y páncreas (8). Histopatológicamente, las células pueden estar arregladas en un patrón trabecular o acinar o la mezcla de ambos. En algunos casos, las células están ubicadas en columnas separadas por sinusoides (9). En áreas diferenciadas, las células tumorales son poliédricas, semejando claramente hepatocitos; puede ser indiferenciado con variación en el tamaño de las células con características tintoriales y contenido citoplasmático como grasa y glicógeno $(4,8)$. Se observa variable cantidad de estroma, grandes espacios vasculares frecuentemente llenos de sangre y ocasionalmente asociados con focos de necrosis neoplásica de hepatocitos. Las células con núcleo central y citoplasma moderadamente eosinofílico, el cual puede ser pálido o vacuolado con glucógeno o lípidos $(4,8)$.

El colangiocarcinoma, es la segunda neoplasia más frecuente del hígado (10). Microscópicamente las células neoplásicas están organizadas en túbulos y acinos, formando islas o cordones, separados por tejido conectivo fibroso; es frecuente observar presencia de mucina en los lúmenes (4).

Los mesoteliomas son neoplasias que se originan de la línea mesotelial de las cavidades celómicas, pleura, peritoneo, pericardio y ocasionalmente de la túnica vaginal testicular; pueden ser localizados, multifocales o difusos $(11,12)$. El mesotelioma maligno es una neoplasia fatal, la cual se ha reportado en humanos y en muchas especies 
animales incluyendo caninos, equinos, bovinos, felinos y ratas $(2,13)$; el sitio más común de presentación es la pleura, seguido por el peritoneo, siendo extremadamente raro hallarlo en pericardio y túnica vaginal testicular (14). Este tumor ha sido reportado en muchas especies incluyendo perros, bovinos, gatos, caballos, ratas y hámster (13).

Los mesoteliomas son clasificados de acuerdo con su patrón histológico en: epitelial, sarcomatoso o bifásico, desmoplásico, micro quístico, de células claras y deciduo (11). El mesotelioma en todas las especies está asociado con la exposición a asbesto, virus Simiam 40 (SV 40) y en algunas ocasiones a óxido de aluminio. Macroscópicamente, se aprecian como nódulos de superficie rugosa (11). Los mesoteliomas benignos y malignos tienen una estructura básica similar, por lo cual en algunos casos puede ser difícil establecer un diagnóstico. Un claro pronóstico de malignidad es la presencia de células tumorales en vasos linfoides a una distancia considerable desde la superficie profunda de los tumores y con metástasis a nódulos linfoides (15). Parece que los vasos sanguíneos y linfáticos del pericardio son los blancos primarios de la enfermedad y el daño de los vasos es la fuente de la efusión $(16,17)$. Se asume que esas células mesoteliales descamadas entran a los vasos linfáticos submesoteliales a través de los poros intercelulares de la serosa y son luego transportados por los nódulos linfáticos que los drenan $(18,15)$.

En los órganos genitales masculinos y femeninos se presenta el tumor venéreo transmisible (TVT), una neoplasia que afecta únicamente a los caninos, localizado primariamente en la mucosa genital externa. Es transmitida sexualmente y los animales con mayor riesgo son perros de 4 a 5 años de edad. Es un tumor altamente contagioso, pero no es zoonótico (19), transmitido por la transferencia de células intactas durante el coito. En animales adultos puede regresar espontáneamente (20).
El objetivo del presente estudio fue clasificar histológicamente y describir las enfermedades neoplásicas en caninos.

\section{MATERIALES Y MÉTODOS}

Se trata de un estudio retrospectivo que se realizócon datos del Laboratorio de Histopatología Veterinaria de Universidad de los Llanos, localizado en la vereda Barcelona del municipio de Villavicencio, departamento del Meta, durante el periodo comprendido entre enero de 2004 y junio de 2007 . Se utilizó la información de los casos clínicos atendidos en la clínica veterinaria de la universidad de los Llanos, así como los resultados de biopsias obtenidas durante procedimientos quirúrgicos y de especímenes procedentes de necropsias, recibidos durante el periodo de estudio en el servicio de diagnóstico de la clínica.

En todos los casos los tejidos fueron procesados siguiendo los protocolos tradicionales de histotecnia de inclusión en parafina, corte y tinción con hematoxilina - eosina. Cada tumor se clasificó según el sitio anatómico de su localización y su histología. En los animales donde se registró más de un tipo de tumor se consideraron como casos separados. En los casos en los cuales el diagnóstico sobre el tipo de tumor fue incierto, se utilizaron coloraciones diferenciales como azul de toloudina y PAS.

Clasificación de las neoplasias. Inicialmente se realizó un estudio descriptivo, con criterio diagnóstico de tipo histopatológico, teniendo en cuenta el diagnóstico preliminar, la localización y la frecuencia de presentación de la neoplasia. Posteriormente, cada caso de neoplasia se caracterizó histológicamente y se clasificó siguiendo los criterios para la clasificación internacional de tumores de los animales domésticos de la organización mundial de la salud (World Health Organization Internacional Histological Classification of Tumors of Domestic Animals. The 
Armed Forces Institute of Pathology. American Registry of Pathology. Washington, DC).

Análisis estadístico. La incidencia de la enfermedad en cada especie y raza, fue estimada a partir del número de casos clasificados como neoplasias, divididos por el total de pacientes de cada categoría, atendidos en el Laboratorio de Histopatología durante el periodo de estudio. Los casos diagnosticados como enfermedad neoplásica fueron clasificados de acuerdo con las variables especie, raza, edad, sexo, órgano afectado, procedencia.

Inicialmente, los datos fueron descritos estadísticamente para establecer las neoplasias más frecuentes en cada especie y el sistema orgánico más afectado. Posteriormente, los datos fueron sometidos a pruebas exactas de Fisher, con el fin de determinar los efectos de la edad, el sexo, la raza y la procedencia del paciente, sobre la presentación de las neoplasias. Todos los procedimientos estadísticos fueron realizados con el software GradPah Instat 3.0 , versión para Windows.

\section{RESULTADOS}

Durante el periodo considerado para el estudio se atendieron un total de 322 casos, de los cuales 113 casos (35\%) fueron diagnosticados como neoplasias; el $82.3 \%$ de las neoplasias (93 de 113 casos) correspondieron a caninos y el porcentaje restante a otras especies. La incidencia anual de diagnóstico de casos de neoplasias durante el periodo de estudio, se ilustra en la figura 1.

Neoplasias cutáneas. Se analizaron 68 protocolos de necropsia, la de mayor proporción fue la especie canina con 56 casos $(82 \%)$, la clasificación se muestra en la tabla 1.

\section{Carcinoma epidermoide o escamocelular. En un paciente con}

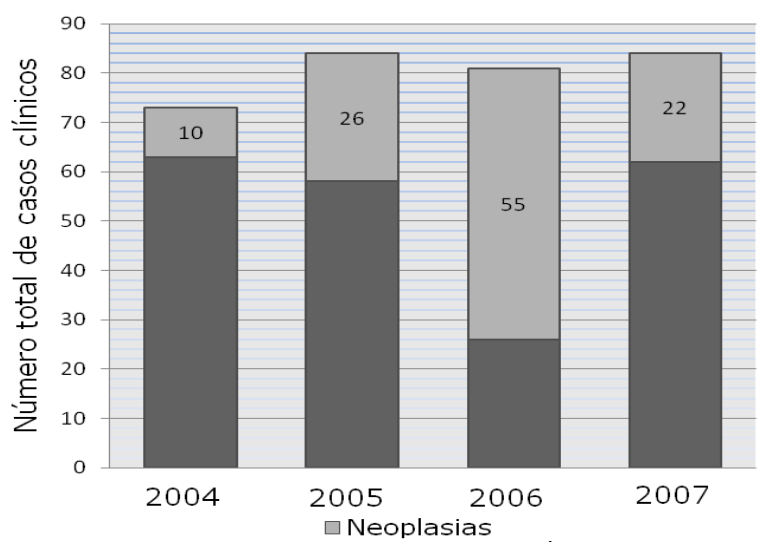

Figura 1. Incidencia de casos de neoplasias entre enero de 2004 y junio de 2007, en el servicio de diagnóstico del Laboratorio de Histopatología de la Clínica Veterinaria de la Universidad de los Llanos, Villavicencio - Meta.

hiperqueratosis en la piel de los miembros posteriores, se observó en todo el tejido la presencia de gran cantidad de islas con centros de queratina y células epiteliales anaplásicas grandes, con gran cantidad de figuras mitóticas (Figura 2A).

Tricoepitelioma. Una masa hallada en el codo del miembro anterior derecho de un paciente se diagnosticó como tricoepitelioma, observándose múltiples quistes de distinto tamaño, cubiertos por epitelio escamoso, llenos de queratina y células fantasma semejando las células del bulbo piloso (Figura 2B).

Mastocitoma canino III. Se encontraron masas móviles en el dorso de un paciente, compatibles con mastocitoma canino grado III, densos cordones de células pleomórficas, con citoplasma indefinido con finos o sin ningún gránulo citoplasmático, núcleo y nucléolo prominentes y con $4-5$ figuras mitóticas por campo (Figura 2C).

Neoplasias de la glándula mamaria. El estudio reveló que de los 13 diagnósticos realizadas de tumores de glándula mamaria, $11(84.6 \%)$ correspondieron a la especie canina, los cuales fueron clasificados como carcinoma simple ( 3 casos), carcinoma complejo (4 casos), 
Tabla 1. Clasificación de las neoplasias del sistema cutáneo, diagnosticadas en caninos.

\begin{tabular}{llc}
\hline \multicolumn{1}{c}{ Origen del tumor } & \multicolumn{1}{c}{ I. Tumores Epiteliales: } & \\
\hline Epidermal & Carcinoma escamocelular & Número de casos \\
\hline \multirow{2}{*}{ Tumor folicular } & Carcinoma de células basales & 15 \\
Sistema melanogénico & Tricoepitelioma & 4 \\
Tumor sebáceo & Acantoma queratinizante infundibular & 3 \\
\hline SUB-TOTAL & Melanoma maligno & 2 \\
\hline & Carcinoma glándula saco anal & 13 \\
\hline Tumor de células de Mast & Mastocitoma & 38 \\
\hline Tumores células en huso & Fibrosarcoma & 11 \\
\hline Tumor histiocítico & Hemangiopericitoma & 1 \\
\hline SUB-TOTAL & Hixosarcoma & 2 \\
\hline TOTAL & & 1 \\
\hline
\end{tabular}

adenocarcinoma ( 2 casos) y carcinosarcoma ( 2 casos).

Carcinoma mamario. En un carcinoma mamario hallado en una hembra canina se observó la formación de túbulos con o sin proyecciones papilares, con hiperplasia alveolar y los alvéolos llenos de material mucinoso en su lumen, las células hipercromáticas, pleomórficas, los núcleos basófilos con nucléolos prominentes (Figura 2D) y marcada proliferación de fibroblastos estromales.

Carcinoma mamario complejo. En este caso una hembra presentó masas múltiples en las mamas. Al examen microscópico se pudo observar un componente de tipo epitelial y mioepitelial. Las células epiteliales muestran patrón túbulo papilar y las células con forma de huso tienen aspecto estrellado. Se presenta metaplasia cartilaginosa en gran parte del tejido, con acompañamiento de células mioepiteliales (Figura 2E), también hay metaplasia escamosa dentro de la neoplasia.

Carcinosarcoma mamario de transición. En una muestra de glándula mamaria de una hembra, la neoplasia está compuesta por células semejando células epiteliales malignas (epiteliales luminales y/o mioepiteliales) y células de tejido conectivo maligno, lo mismo que mezcla de los dos tipos de componentes carcinomatosos. En el tejido se aprecia el material mucoide y una porción condrosarcomatosa, con la presencia de células epiteliales malignas (Figura 2F).

Neoplasias del sistema digestivo. De 13 neoplasias de órganos digestivos analizadas, $8(61.5 \%)$ correspondieron a caninos, cuya clasificación se muestra en la tabla 2. 

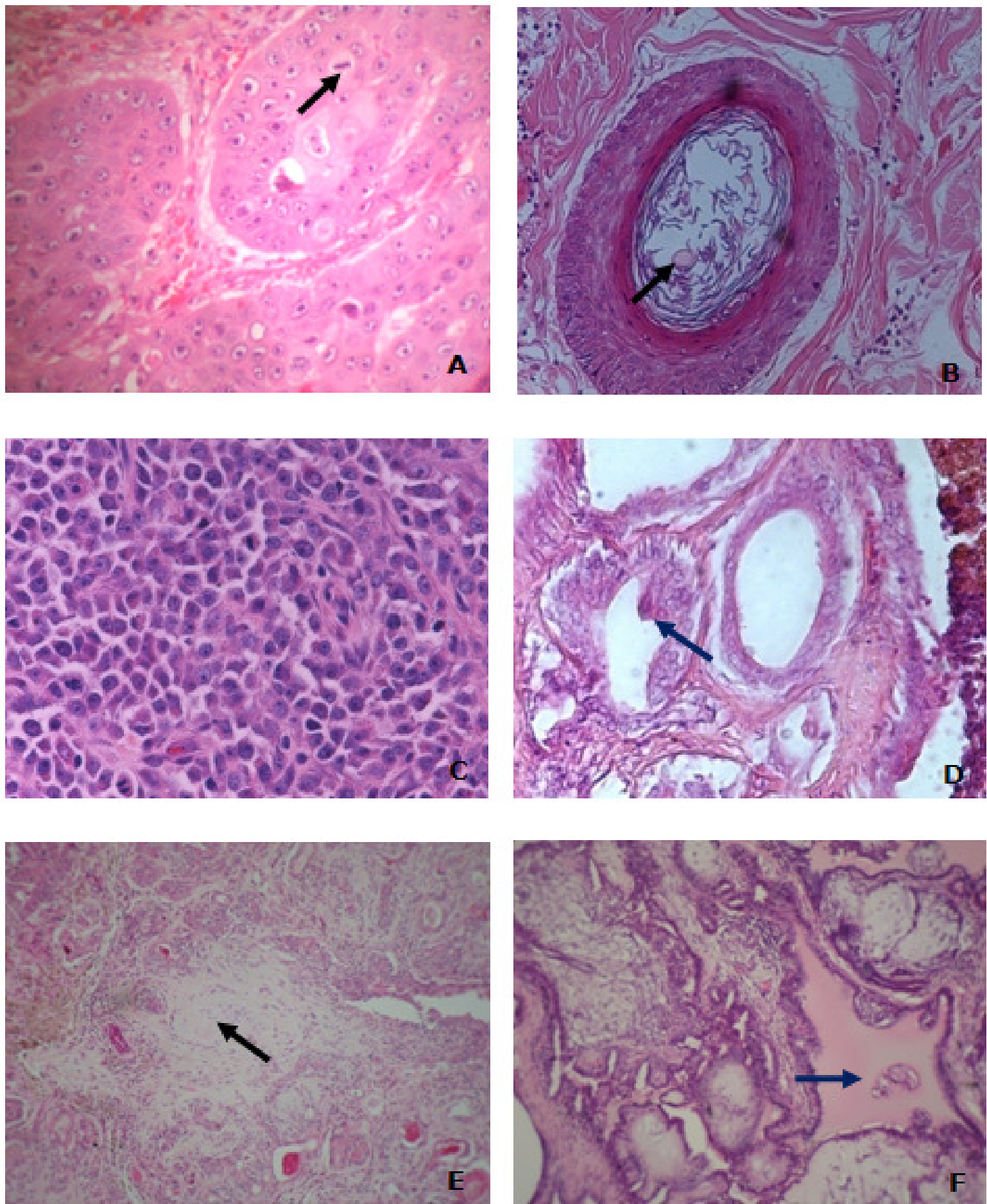

Figura 2. Neoplasias del sistema cutáneo. Carcinoma epidermoide (H-E, 40x) A, con figura de mitosis $(\rightarrow)$. Tricoepitelioma $(H-E, 20 X) B$, quiste córneo con células fantasma $(\rightarrow)$. Mastocitoma canino grado III $(H-E, 40 x)$ C. Carcinoma mamario (H-E, 40X) D, alvéolos con hiperplasia de células cuboides con proyecciones papilares $(\rightarrow)$. Carcinoma complejo de glándula mamaria (H-E, 10X) E, con metaplasia de tipo cartilaginosa $(\rightarrow)$. Carcinosarcoma mamario de transición $(H-E, 40 X)$, porción carcinomatosa, condrosarcomatosa y material mucoide $(\rightarrow)$. 
Tabla 2. Clasificación de las neoplasias halladas en el sistema digestivo de caninos

\begin{tabular}{lcc}
\hline \multicolumn{1}{c}{ Origen del tumor } & $\begin{array}{c}\text { I. Tumores Epiteliales: } \\
\text { Tipo de tumor }\end{array}$ & Número de casos \\
\hline Epitelio ductual biliar & Colangiocarcinoma & 1 \\
Hígado & Hepatocolangiocarcinoma & 1 \\
Epitelio odontogénico & Carcinoma Hepatocelular & 1 \\
Mesotelio peritoneal & Ameloblastoma & 1 \\
Sistema Melanogénico (metástasis) & Melanoma Maligno & 1 \\
\hline SUB-TOTAL & & 1 \\
\hline & II. Tumores Mesenquimales: & 6 \\
\hline Vasos sanguíneos & Hemangiosarcoma & 1 \\
Tumor hematopoyético & Linfoma & 1 \\
SUB-TOTAL & & 2 \\
\hline TOTAL & & 8 \\
\hline
\end{tabular}

Hemangiosarcoma. En un paciente se encontró una masa en la superficie del bazo llena de líquido amarillento, la cual se diagnosticó como hemangiosarcoma por la presencia marcada de canales vasculares completos e incompletos llenos de eritrocitos y alrededor de los canales zonas hemorrágicas (Figura 3A). Se muestra la neoplasia (Figura 3B).

Neoplasias del aparato genital. Neoplasias afectando el aparto genital de la hembra se analizaron en total 8 casos, 6 de los cuales correspondieron a caninas. En machos caninos fueron diagnosticados 8 casos, cuya clasificación y localización se muestra en la Tabla 3.

Tumor venéreo transmisible. En un canino macho se halló una masa sólida en la región abdominal derecha la cual se diagnosticó como tumor venéreo transmisible, donde se ve una densa población de células redondas u ovoides, relación núcleo-citoplasma mínimo (Figura 4A). Citoplasma pálido con aspecto granulado fino y vacuolas, núcleos redondos con agregados cromatínicos densos. Figuras mitóticas aberrantes (Figura 4B).

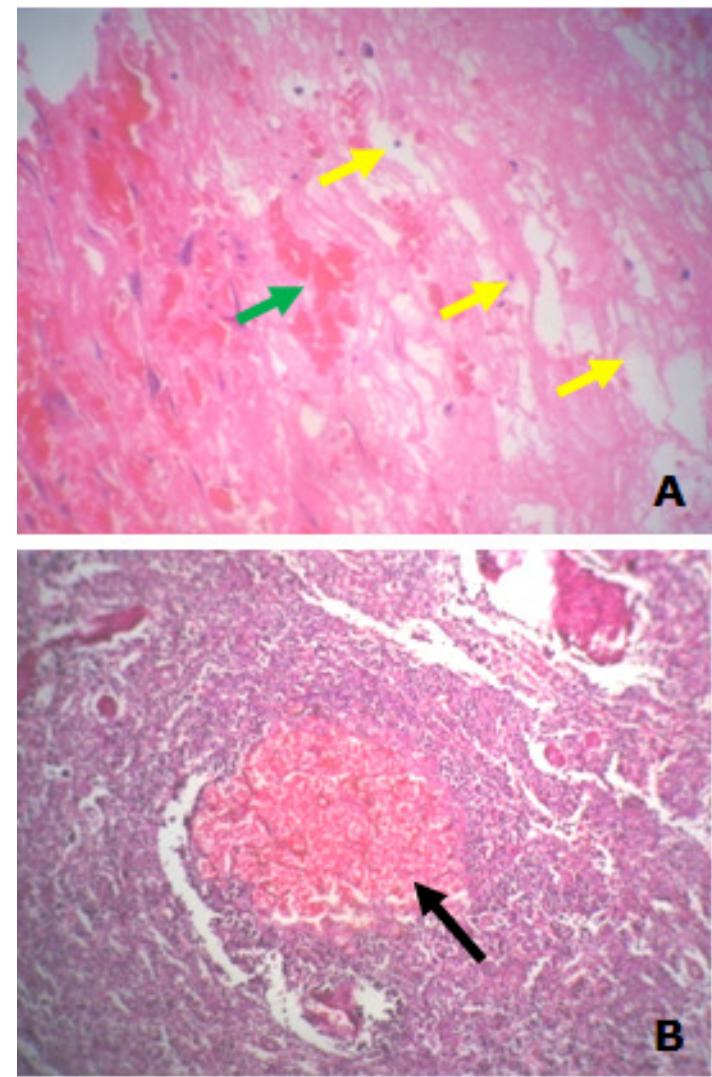

Figura 3. Hemangiosarcoma (H-E, 10X y 20X). A. Canales vasculares completos e incompletos $\rightarrow$ con abundante contenido de eritrocitos $(\rightarrow)$. B. Hemangiosarcoma en bazo $(\rightarrow)$. 
Tabla 3. Clasificación y localización de las neoplasias diagnosticadas en el aparato genital de caninos.

\begin{tabular}{lcc}
\hline \multicolumn{1}{c}{ Tipo de tumor } & Localización & $\begin{array}{c}\text { Número } \\
\text { de casos }\end{array}$ \\
\hline HEMBRA: & & \\
\hline Leiomiosarcoma & Vulva & 1 \\
Tumor Células & Ovario & 1 \\
Granulosa & Ovario & 1 \\
Luteoma & Útero & 1 \\
Hemangiosarcoma & Vagina & 2 \\
TVT & & 6 \\
\hline Subtotal & & \\
\hline MACHO: & Pene y & 5 \\
\hline TVT & prepucio & 2 \\
Tumor células de & Testículo & 1 \\
Sertoli & Testículo & 8 \\
Seminoma & & 14 \\
\hline Subtotal & & \\
\hline ToTAL & & \\
\hline
\end{tabular}

La incidencia de enfermedades neoplásicas en caninos, agrupada por raza, sexo, edad y procedencia se presenta en la tabla 4.

Tabla 4. Número de casos y porcentaje de individuos de la especie canina afectados por enfermedades neoplásicas, agrupados según la raza, sexo, edad y procedencia del paciente.

\begin{tabular}{|c|c|c|c|}
\hline \multicolumn{3}{|c|}{ ENFERMEDAD NEOPLÁSICA } & \multirow{2}{*}{$\begin{array}{c}\text { Total de } \\
\text { Casos }\end{array}$} \\
\hline & SI & No & \\
\hline \multicolumn{4}{|l|}{ RAZA: } \\
\hline Mestiza & $48(26 \%)$ & $30(17 \%)$ & $78(43 \%)$ \\
\hline Otras razas & $45(25 \%)$ & $58(32 \%)$ & $103(57 \%)$ \\
\hline \multicolumn{4}{|l|}{ SEXO: } \\
\hline Macho & $61(34 \%)$ & $45(25 \%)$ & $106(59 \%)$ \\
\hline Hembra & $32(18 \%)$ & $43(23 \%)$ & $75(41 \%)$ \\
\hline \multicolumn{4}{|l|}{ EDAD: } \\
\hline $0-4$ años & $22(12 \%)$ & $61(34 \%)$ & $83(46 \%)$ \\
\hline$>4$ años & $71(49 \%)$ & $27(15 \%)$ & $98(54 \%)$ \\
\hline \multicolumn{4}{|c|}{ PROCEDENCIA: } \\
\hline Urbana & $66(36 \%)$ & $28(16 \%)$ & $94(52 \%)$ \\
\hline Rural & $27(15 \%)$ & $60(33 \%)$ & $87(48 \%)$ \\
\hline TOTAL & $93(51 \%)$ & $88(49 \%)$ & $181(100 \%)$ \\
\hline
\end{tabular}

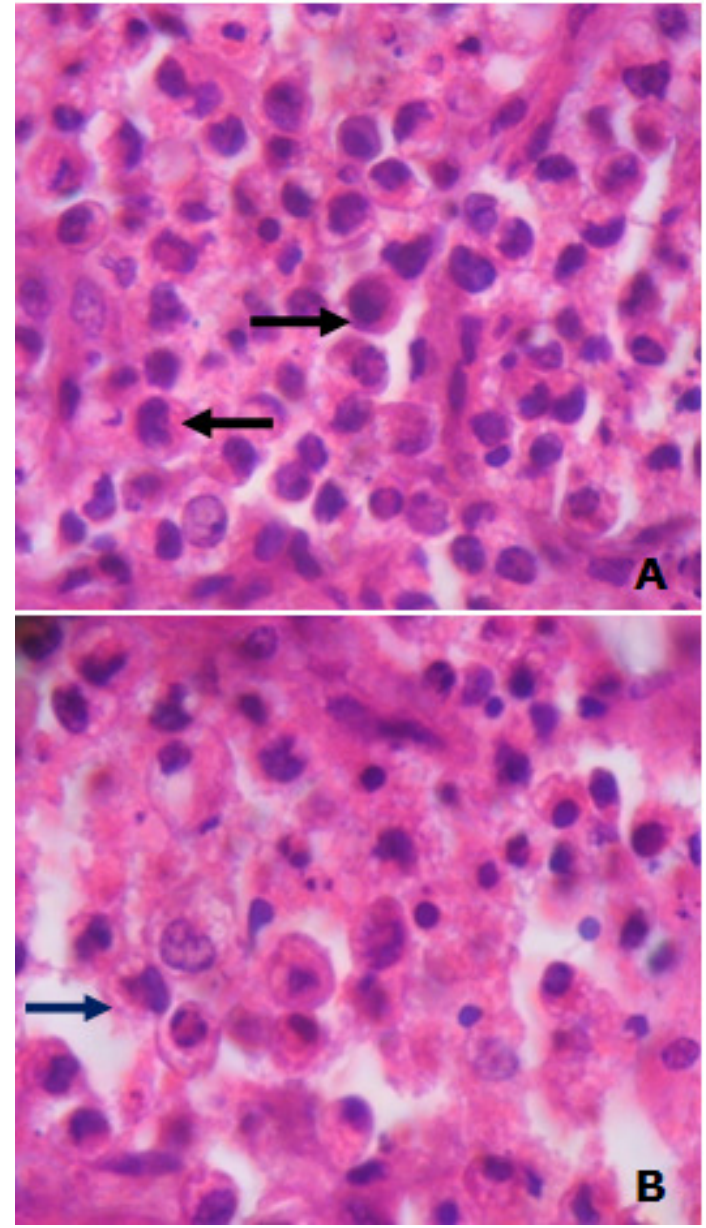

Figura 4. Tumor venéreo transmisible (H-E, 100x). A. Células ovoides o redondas, núcleo redondo, agregados cromatínicos densos, $(\rightarrow)$ relación núcleo citoplasma mínima. B. citoplasma pálido con aspecto granulado fino y vacuolas $(\rightarrow)$.

\section{DISCUSIÓN}

La especie canina resultó ser la más afectada, lo cual puede explicarse por el hecho que los caninos constituyen la especie con mayor número de pacientes atendidos en la Clínica Veterinaria de la Universidad de los Llanos. Esta consideración resulta importante puesto que todos los casos estudiados en este trabajo procedían de dicho Centro de Diagnóstico; no obstante, estos hallazgos coinciden con un estudio de la Universidad de Antioquia, en el cual la especie canina también resultó ser la más afectada $(56.5 \%)$, según lo reportado por Ferreira 
de la Cuesta y Pedraza (21). De igual forma, Zaldívar et al (22), hallaron una mayor incidencia en la especie canina.

Se diagnosticaron 68 casos de tumores del sistema cutáneo, siendo la especie canina la más afectada ( $81 \%)$, lo cual puede estar relacionado con la mayor facilidad para detectar este tipo de neoplasias, comparadas con aquellas localizadas en sitios más profundos, que sólo se diagnostican postmortem (21). La especie canina fue la más afectada por carcinoma epidermoide (56.5\%), el cual constituyó el de mayor presentación, seguido del tricoepitelioma, mastocitoma, carcinoma de células basales, histiocitoma y acantoma. Estos resultados sugieren una alta incidencia del carcinoma de células escamosas y de tricoepiteliomas del sistema cutáneo, pudiendo estar relacionados con la ubicación de Colombia en el trópico, donde es alta la incidencia de radiaciones UV (23). Esto coincide con lo observado por Zaldívar et al (22). Asimismo, Torres Vidales (24) evaluó 497 casos de tumores de piel en caninos atendidos en el Laboratorio de Patología Veterinaria de la Universidad Nacional, reportando que las neoplasias de origen epitelial más comunes fueron: carcinoma escamocelular $(29 \%)$, tumor de células basales $(20 \%)$, adenoma de células hepatoides $(19 \%)$. Sin embargo, otros estudios no hallaron relación entre la exposición a radiación solar con el desarrollo de carcinoma escamo celular y tricoepitelioma $(21,25)$.

Las razas de perros más afectadas fueron la mestiza, Bóxer, Labrador, French Poodle y Pastor Alemán; lo cual coincide con las observaciones de Ferreira de la Cuesta y Pedraza (21), quienes encontraron mayor incidencia en caninos de las razas mestizas y Pastor Alemán.

De los 13 diagnósticos de tumores de glándula mamaria, 11 casos ( $84.6 \%)$, correspondieron a hembras caninas. Este porcentaje coincide con el reportado por Ferreira de la Cuesta y Pedraza (21), quienes informaron una incidencia del $82.5 \%$. De la misma manera, Rostami et al (26) en un estudio de 10 años en la
Universidad de Miyazaki, sobre incidencia de tumores en animales domésticos, reportaron que las neoplasias más comunes en pequeños animales eran las de glándula mamaria. En el caso de los caninos, todas las neoplasias diagnosticadas fueron malignas, siendo las más frecuentes las epiteliales (38.5\%), lo cual concuerda con el estudio de la Universidad de Antioquia, en el cual se reporta que la neoplasia más frecuente fue el carcinoma, representando un $58.6 \%$ de los casos. Asimismo, se corrobora lo reportado por Torres (27), quien evaluó 173 casos de tumores de glándula mamaria del archivo del Laboratorio de Patología de la Universidad Nacional, hallando que el $59 \%$ de los casos correspondieron a neoplasias malignas.

Las razas más afectadas con tumores mamarios fueron la mestiza, seguida de French Poodle, Chow Chow y Labrador, lo cual coincide con lo reportado por Ferreira de la Cuesta y Pedraza (21), quienes informaron mayor presentación de neoplasias en la raza mestiza seguida por Pastor Alemán, Cocker Spaniel Americano, Pequinés, Pinsher, Poodle y Doberman. Por su parte, Torres y Eslava (28) reportaron que existe cierta concordancia respecto a la posible predisposición de razas como Springer Spaniels, Labrador, Cocker Spaniel, Pointer, Pastor Alemán, French Poodle, Maltes, Yorkshire y Dachshunds. También Torres Vidales (24) reportó que las razas que presentan más frecuentemente tumores mamarios son Cocker Spaniel $(22,8 \%)$, French Poodle (19\%) y mestiza $(14,5 \%)$.

Los resultados muestran una mayor prevalencia de neoplasias malignas de la glándula mamaria en los caninos (84.6\%), coincidiendo con Kelsey et al (23), quienes reportaron que las hembras caninas tuvieron mayor presentación de neoplasias malignas de glándula mamaria ( $51 \%)$. Además, siempre fueron animales enteros los más afectados, lo cual permitiría inferir que el uso de algunos progestágenos para la esterilización canina, podría ser un factor predisponente $(6,24,29)$. Sin embargo, Kesley et al (23) sugieren que la castración a edad temprana protege contra neoplasias de glándula mamaria. La 
presencia de receptores para estrógenos y progesterona en tumores mamarios en perros, sugiere soporte hormonal.

En el sistema digestivo de caninos se diagnosticaron 8 neoplasias de un total de $13(61.5 \%)$ lo cual es similar a lo reportado por Ferreira de la Cuesta y Pedraza (21), donde los caninos presentaron una prevalencia de 54.7\%. En el presente estudio, las de mayor presentación fueron las neoplasias hepáticas $62.5 \%$ ( 5 de 8 casos). Estos resultados son similares a los reportados por Zaldívar et al (22), pero no concuerdan con el estudio de la Universidad de Antioquia, en el que se halló mayor incidencia de neoplasias de las glándulas perianales (54.6\%), seguido de las hepáticas $(22.3 \%)(21)$, también es diferente al reporte del Reino Unido, en el cual se presentó mayor incidencia de Hemangiosarcoma del tracto digestivo (25).

En este estudio se observó que las razas más afectadas fueron la mestiza, Golden Retriever, Samoyedo y Pastor Alemán, presentándose un sólo caso de mesotelioma peritoneal. En un estudio epidemiológico en perros, realizado por Kelsey et al (23), basado en 18 casos con confirmación histológica de mesotelioma, un $33 \%$ de ellos fue en el peritoneo, $28 \%$ en la pleura, $28 \%$ en peritoneo y pleura y $11 \%$ en pericardio. De los 18 perros con mesotelioma 17 fueron machos, únicamente un caso ocurrió en un perro de menos de 5 años de edad (23). En el presente estudio el mesotelioma del perro es de tipo epitelioide y bifásico comprometiendo pleura, pericardio, mediastino, peritoneo, mesenterio. La historia del paciente está relacionada con la exposición a asbesto por inhalación, puesto que el animal vivía en un taller de remaches de bandas de frenos. En este contexto, Yamate et al (12) reportaron el hallazgo de fibras de crisolitos de asbesto en tejidos pulmonares de perros con mesotelioma maligno.

En el aparato genital de la hembra se analizaron 7 tumores, de los cuales 6 casos se presentaron en caninos $(85 \%)$, siendo el Tumor Venero Transmisible (TVT) la neoplasia más frecuente, lo cual coincide con el estudio de la Universidad de Antioquia.

En el tracto genital de machos caninos se hallaron 8 neoplasias, de las cuales 5 correspondieron a TVT (62\%), dos a tumor de células de Sertoli y una a seminoma, coincidiendo con los estudios realizados en Cuba por Kelsey et al (23), donde los tumores de células de Sertoli, seminomas y tumores de células intersticiales fueron los más frecuentes. Lo anterior es similar a los reportes de Ferreira de la Cuesta y Pedraza (21), quienes hallaron incidencia de seminoma de $55 \%$ y de TVT de $58 \%$.

La neoplasia más frecuente reportada por Dobson et al (25) Ferreira de la Cuesta y Pedraza (21) en perros es el seminoma, ocupando el primer lugar de presentación en el Reino Unido y el segundo lugar en Antioquia, estos resultados parecen estar relacionados con la mayor exposición de este tejido de revestimiento a la acción adversa del ambiente de agentes virales cancerígenos; también por la sensibilidad del tejido epitelial y por la propiedad de multiplicación de sus células (22).

Al evaluar la posibilidad de hallar relación entre la raza y la presentación de neoplasias, se identificó que animales mestizos tendrían mayor predisposición. Sin embargo, Ferreira de la Cuesta y Pedraza (23) reportaron mayor presentación de neoplasias de glándula mamaria en perras de razas puras.

Con los datos obtenidos se estableció una relación entre el sexo en los caninos y la predisposición a los tumores. Entre los 181 casos analizados, a pesar de hallar 106 machos y 75 hembras y una incidencia de $34 \%$ y $18 \%$, respectivamente, el efecto del sexo no fue significativo.

El efecto de la edad sobre la incidencia de los tumores en caninos reveló un efecto significativo, ya que la incidencia de estas patologías en individuos mayores de 4 años fue mayor. Este resultado es similar al reportado por Dobson et al (25) en el Reino Unido, donde hallaron que la mayoría de los tumores ocurren en perros mayores de nueve años. 
La relación entre la presentación de neoplasias y la procedencia del paciente también fue altamente significativa, mostrando que los caninos provenientes de la ciudad están más predispuestos a la presentación de neoplasias; no obstante, este resultado puede sugerir que los propietarios de estos animales son más conscientes y tienen relativamente mayor posibilidad de llevarlos a los centros de diagnóstico.

La relación entre la procedencia de los animales y la presentación de neoplasias revelada en este trabajo, implica la necesidad de realizar otros trabajos, que permitan determinar los factores ambientales que puedan estar implicados, tales como nutrición, uso de aditivos y conservantes en los concentrados, contaminación de los mismos con micotoxinas, dioxinas, presencia de grasas rancias, compuestos nitrogenados, etc., los cuales podrían actuar como factores iniciadores y promotores de procesos neoplásicos.

En conclusión, los tumores de piel fueron los de mayor incidencia en caninos, siendo el carcinoma escamocelular la neoplasia más frecuente, afectando preferentemente a animales viejos mestizos, mayores de 9 años de edad.

\section{REFERENCIAS}

1. Kitchell B, Manfra S. Oral Tumors in Dogs and Cats. Part I. Diagnosis and Clinical signs. Compendium 1998; 20(9):1011-1020.

2. Baer KE, Helton K. Multicentric squamous cell carcinoma in situ resembling Bowen's disease in cats. Vet Pathol 1993; 30:535-543.

3. Mcgavin D, Carlton W, Zachary J. Thomson's Special Veterinary Pathology. 3 ed. Philadelphia - USA: Mosby Elsevier, 2001.

4. Meuten DJ (ed). Tumors in domestic Animals. 4 ed. Ames - USA: Iowa State press; 2002.

5. Sueiro CR, Daleck AC, Alessi A. Ultraestrutura dos mastócitos de diferentes tipos histológicos de mastocitoma em cães. Arq Bras Med Vet Zootec 2002; 54(3):255-258.

6. Torres G, y Eslava P. Mastocitoma Canino. Orinoquia 2006; 10(2):45-51.

7. RechI R, Graça D, Kommers GS, Raffi M, Garmatz S. Mastocitoma cutâneo canino: estudo de 45 casos. Arq Bras Med Vet Zootec 2004; 56(4):441-448.
8. Jeong W, Do S, Sohn M, Yun H, Kwon $O$, et al. Hepatocellular Carcinoma with Metastasis to the Spleen in a Holstein Cow. Vet Pathol 2005; 42(2):230-2

9. Zhang S, Cong W, Wu M. Focal nodular hyperplasia with concomitant hepatocellular carcinoma: a case report and clonal analysis. J Clin Pathol 2004; 57:556-559.

10. Salas AA, Gonzailez AL, Goepfert RH. Combined hepato-cholangiocarcinoma. Case presentation and literature review. Departamento de Patologiìa, Instituto Nacional de Cancerologiìa, Meixico, D.F. Rev Gastroenterol Mex 2006; 71(4): 483-486.

11. Morini M, Bettini G, Morandi F, Burdisso $R$, Marcato P. Deciduoid Peritoneal Mesothelioma in a Dog. Vet Pathol 2006; 43: 198-201.

12. Yamate J, Tomita A, Kuwamura M, Mitsunaga F, Nakamura S. Spontaneus peritoneal malignat mesothelioma in a geriatric Japanese macaque (Macaca fuscata). Exp Anim 2007; 56(2):155159. 
13. Bacci B, Morandi F, De Meo M, Marcato P. Ten cases of Feline Mesothelioma: an Inmunohistochemical and ultraestructural study. J Comp Pathol 2006; 134: 347- 354 .

14. Abbas A, Peter $H$, Wunsch. Epithelioid and sarcomatoid malignant pleural mesothelioma in endoscopio gastric biopsies: A diagnostic pitfall. Pathol Res Pract 2006; 202: 617-622.

15. Peters M, Tenhundfeld I, Stephan I, Hewicker T. Embolized Mesothelial cells within Mediastinal Lymph Nodes of three dogs with idiopathic haemorrhagic pericardial effusion. J Comp Path 2002; 128: 107-112.

16. Berg R, Wingfield $W$, Hoopes $P$. Idiopatic hemorrhagic pericardial effusion in eight dogs. J Am Vet Med Assoc 1984; 185: 988-992.

17. Machida N, Tanaka R, Takemura N, Fujii Y, Ueno A, Mitsumori K. Development of Pericardial Mesothelioma in Golden Retrievers with a long- term History of Idiopathic Haemorrhagic Pericardial Effusion. J Comp Pathol 2004; 131:166-175.

18. Suarez Vilela D, Izquierdo Garcia F. Embolization of mesothelial cells in lymphatics: the route to mesothelial inclusions in lymph nodes? Histopathol 1998; 33:570-575.

19. Rogers K. Transmissible Venereal Tumor. Compendium on Continuing Education for the Practicing Veterinarian. 1997; 19(9):1036-1045.

20. Santos FHD, Vasconcelos DC, Moro L, Nunes JES, Paixao TA. Apoptose no tumor venéreo transmissível canino: características morfológicas e evidenciação bioquímica. Arq Bras Med Vet Zootec. 2001; 53(5):557-562.

21. Ferreira de la Cuesta G, Pedraza F. Patología Veterinaria 1 ed. Medellín, Colombia: Universidad de Antioquia; 2003. p 622.
22. Zaldívar $N$, Puebla $H$, Ferrales $Y$, Almaguer Y, Vaillant Y. Importancia de las neoplasias en los animales domésticos. Neoplasias diagnosticadas en los centros de epizootiología y diagnóstico veterinario de Guantánamo y Granma, Cuba. Rev Prod Anim 2002; 14:51-53.

23. Kelsey J, Antony S. Moore A, Glickman L. Epidemiologic studies of risk factors for cancer in pet dogs. Epidemiol Rev $1998 ; 20(2): 204-217$.

24. Torres-Vidales G. Estudio histológicoretrospectivo de tumores en caninos diagnosticados en el laboratorio de patología de la Facultad de Medicina Veterinaria de la Universidad Nacional de Colombia. [Tesis de especialización] Bogotá: Universidad Nacional de Colombia, Departamento de Ciencias para la Salud Animal, Facultad de Medicina Veterinaria y Zootecnia; 2003.

25. Dobson J, Samuel S, Milstein H, Rogers, Wood J. Canine neoplasia in the UK: estimates of incidence rates from a population of insured dogs. J Small Anim Pract 2002; 43:240-246.

26. Rostami M, Tateyama S, Uchida K, Naitou, H. Yamaguchi, R. Otsuka, H. Tumors in domestic animals examined during a ten-year period (1980 to 1989) at Miyazaki University. J Vet Med Sci 1994; 56(2): 403-405.

27. Torres Vidales G; Botero Espinosa L. A restrospective histopathological study of mammary gland neoplasia in canines (1975-2000). Orinoquia 2008; 12(1):80-88.

28. Torres G, Eslava P. Tumores mamarios en caninos: Adenocarcinoma complejo de glándula mamaria con metástasis a ganglio linfático regional. Reporte de caso. Rev Orinoquia 2007; 11(1): $99-110$.

29. Sorenmo K. Canine mammary gland tumors. Vet Clin North Am Small Anim Pract 2003; 33:573-596. 